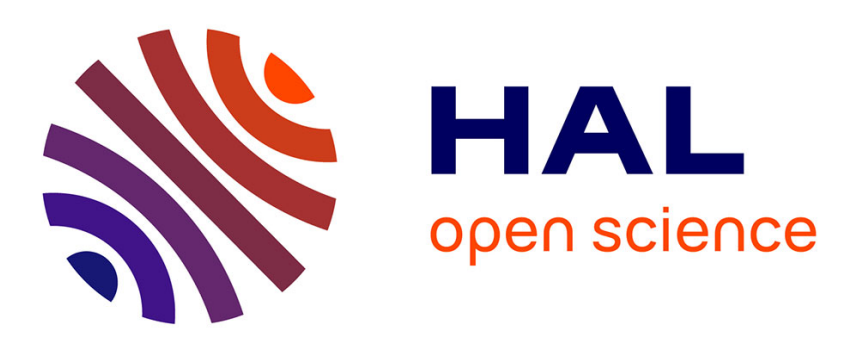

\title{
Strain induced Pockels effect in silicon for electro-optic modulation
}

\author{
Christian Lafforgue, Mathias Berciano, Lucas Deniel, Guillaume Marcaud, \\ Xavier Le Roux, Carlos Alonso-Ramos, Daniel Benedikovic, Vladyslav \\ Vakarin, Alicia Ruiz-Caridad, Paul Crozat, et al.
}

\section{To cite this version:}

Christian Lafforgue, Mathias Berciano, Lucas Deniel, Guillaume Marcaud, Xavier Le Roux, et al.. Strain induced Pockels effect in silicon for electro-optic modulation. Photonics West, Feb 2020, San Francisco, United States. 10.1117/12.2545682 . hal-02933344

\section{HAL Id: hal-02933344 https://hal.science/hal-02933344}

Submitted on 8 Sep 2020

HAL is a multi-disciplinary open access archive for the deposit and dissemination of scientific research documents, whether they are published or not. The documents may come from teaching and research institutions in France or abroad, or from public or private research centers.
L'archive ouverte pluridisciplinaire HAL, est destinée au dépôt et à la diffusion de documents scientifiques de niveau recherche, publiés ou non, émanant des établissements d'enseignement et de recherche français ou étrangers, des laboratoires publics ou privés. 


\title{
Strain induced Pockels effect in silicon for electro-optic modulation
}

\author{
Christian Lafforgue $^{\mathrm{a}}$, Mathias Berciano ${ }^{\mathrm{a}, 1}$, Lucas Deniel ${ }^{\mathrm{a}}$, Guillaume Marcaud ${ }^{\mathrm{a}, 2}$, Xavier Le \\ Roux $^{\mathrm{a}}$, Carlos Alonso-Ramos ${ }^{\mathrm{a}}$, Daniel Benedikovic ${ }^{\mathrm{a}}$, Vladyslav Vakarin ${ }^{\mathrm{a}, 3}$, Alicia \\ Ruiz-Caridad $^{\mathrm{a}}$, Paul Crozat ${ }^{\mathrm{a}}$, Delphine Marris-Morini ${ }^{\mathrm{a}}$, Eric Cassan ${ }^{\mathrm{a}}$, and Laurent Vivien ${ }^{\mathrm{a}}$ \\ a Université Paris-Saclay, CNRS, Centre de Nanosciences et de Nanotechnologies, 91120, \\ Palaiseau, France \\ ${ }^{1}$ Now at IMEC, Kapeldreef 75, Leuven, Belgium \\ ${ }^{2}$ Now at Yale University, New Haven, Connecticut, USA \\ ${ }^{3}$ Now at Nexdot, 93230 Romainville, France
}

\begin{abstract}
The strong evolution of silicon photonics towards very low power consumption circuits leads to the development of new strategies for photonic devices, especially for power-hungry components such as optical modulators. One strategy is to use Pockels effect in $\mathrm{Si}$ waveguides. However, bulk $\mathrm{Si}$ is a centrosymmetric semiconductor, which cannot exhibit any second order optical nonlinearities. Nonetheless, under a strain gradient, generated by depositing a stressed layer on $\mathrm{Si}$ waveguides, this restriction vanishes. In our work, we experimentally demonstrated a Pockels effect based electro-optic modulation at high frequency $(>5 \mathrm{GHz})$ using a strained silicon Mach-Zehnder modulator.
\end{abstract}

Keywords: Silicon photonics, Pockels effect, electro-optic modulation

\section{INTRODUCTION}

For the last decades, the demand in data transmission has increased exponentially. This has led to an enormous power consumption due to electrical interconnects in datacenters and cooling systems for electronic devices. A solution to paliate this problem is to use photonics. Indeed, photonics devices offer high bandwidths and require less energy than electronic devices. However, photonics based functionalities are often implemented with materials that are not compatible with current industrial fabrication processes. Since the microelectronics industry is based on silicon, it appears to be clear that this material is interesting for the development of photonic integrated circuits. Silicon photonics is then being considered as the future photonic platform for low power consumption optical communications. ${ }^{1}$ Numerous demonstrations of optoelectronic devices have been reported including plasma dispersion effect based optical modulators, ${ }^{2,3}$ germanium photodetectors ${ }^{4,5}$ or IIIV laser on silicon $(\mathrm{Si})$ platform. ${ }^{6,7}$ Nevertheless, the strong evolution of silicon photonics towards very low power consumption circuits call upon new strategies to be developed, especially for power-hungry components such as optical modulators. One strategy is to use Pockels effect in Si waveguides. However, bulk Si is a centrosymmetric semiconductor, which cannot exhibit any second order optical nonlinearities. Nonetheless, under a strain gradient, generated by depositing a stressed layer, typically by silicon nitride (SiN) on Si waveguides, this restriction vanishes. ${ }^{8-10}$ Over the years, many attempts to characterize the second order nonlinear susceptibility tensor through Pockels effect have been performed. However, the semiconductor nature of Si hinders correct analysis. Indeed, carriers in $\mathrm{Si}$, at the $\mathrm{Si} / \mathrm{SiN}$ interface and in $\mathrm{SiN}$ induce a refractive index change due to plasma dispersion effect and a screening of the electric field when performing electro-optic modulation. Studies have shown that at low frequency $(<5 \mathrm{GHz})$, the observed electro-optic modulation comes mainly from these charge effects. ${ }^{11,12}$ In our work, we experimentally demonstrated an unambiguous Pockels effect based electro-optic modulation at high frequency $(>5 \mathrm{GHz})$ using a silicon Mach-Zehnder modulator. ${ }^{13}$

christian.lafforgue@c2n.upsaclay.fr 


\section{ELECTRO-OPTIC EFFECT ORIGINS}

In order to achieve electro-optic modulation, several effects can be exploited. Among them, we can cite Pockels effect, which is a second order nonlinear optics effect, and plasma dispersion effect, which comes from changes of free carriers concentrations in the device.

\subsection{Pockels effect}

It is well known that Pockels effect cannot occur in a centrosymmetric material since it is a second order effect. Silicon being a centrosymmetric crystal, a solution that have been studied is to stress a silicon waveguide in such a way that a heterogeneous strain appears to break the crystal symmetry, as shown in the schematic of figure 1.
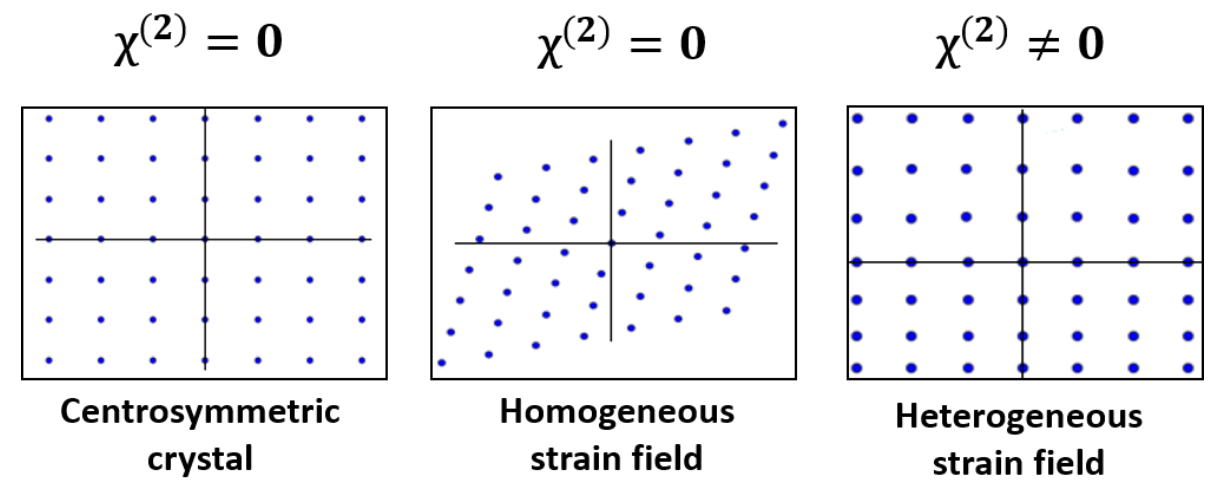

Figure 1. Schematic 2D representation of the effect of strain on the second order susceptibility of a crystal.

By calculating the strain dependent energy bonds between atoms in the crystal lattice, it is possible to establish a link between the second order dielectric polarization and the strain gradients. The second order susceptibility tensor $\chi^{(2)}$ is then linked to the strain gradients in the device as follows: ${ }^{8}$

$$
\chi_{i j k}^{(2)}=\sum_{l m n} \Gamma_{i j k, l m n} \eta_{l m n}
$$

where $i, j, k, l, m, n$ denotes spatial orientations, $\Gamma$ is a $6^{t h}$ order tensor depending only on the material and the crystalline orientation, $\eta$ is a $3^{r d}$ order tensor describing the strain gradients.

The effective index change $\Delta n_{e f f, p}$ is then given by :

$$
\Delta n_{e f f, p}=2 c \varepsilon_{0} \frac{\int_{w g}\left(\chi^{(2)} \cdot \mathbf{F}\right)|\mathbf{E}|^{2} d S}{\int_{\infty}\left(\mathbf{E} \times \mathbf{H}^{*}+\mathbf{E}^{*} \times \mathbf{H}\right) \cdot \mathbf{z} d S}
$$

with $\mathbf{E}(\mathbf{H})$ the optical electric (magnetic) field distribution, $\mathbf{F}$ the driving electric field, $w g$ denoting the waveguide cross section, and $\mathbf{z}$ the dimension along the propagation of the optical mode.

\subsection{Plasma dispersion effect}

In a semiconductor material, free carriers are able to move under the action of an electric field. The free carriers concentration changes are responsible of a change of refractive index $\Delta n$ and a change of propagation loss $\Delta \alpha$ as described by the Soref-Bennett equations for silicon at $1550 \mathrm{~nm}$ wavelength: ${ }^{2}$

$$
\begin{gathered}
\Delta n=-\left(8.8 \cdot 10^{-22} \Delta N_{e}+8.5 \cdot 10^{-18} \Delta N_{h}^{0.8}\right) \\
\Delta \alpha=6.5 \cdot 10^{-18} \Delta N_{e}+6 \cdot 10^{-18} \Delta N_{h}
\end{gathered}
$$

where $\Delta N_{e}$ and $\Delta N_{h}$ are the change of concentration of electrons and holes respectively (in $\mathrm{cm}^{-3}$ ). 
The change of effective index $\Delta n_{e f f, c}$ is then given by equation 5 :

$$
\Delta n_{e f f, c}=2 c \varepsilon_{0} n \frac{\int_{w g} \Delta n|\mathbf{E}|^{2} d S}{\int_{\infty}\left(\mathbf{E} \times \mathbf{H}^{*}+\mathbf{E}^{*} \times \mathbf{H}\right) \cdot \mathbf{z} d S}
$$

\subsection{Total effect}

In a non-centrosymmetric semiconductor, both Pockels effect and plasma carriers effect play a role on the effective index change, giving:

$$
\Delta n_{e f f, t o t a l}=\Delta n_{e f f, p}+\Delta n_{e f f, c}
$$

Many studies of strain induced Pockels effect in silicon have been published, but most of them neglected the plasma dispersion effect, leading to a large overestimation of the strain induced $\chi^{(2)}$. Moreover, additionally to th effective index change they induce, the free carriers displacements tend to reduce the total driving electric field in the waveguide, the latter being lower than the driving field predicted without taking into account those free carriers. This screening effect then reduces the strain-induced Pockels effect, as shown on the schematic of figure 2 .

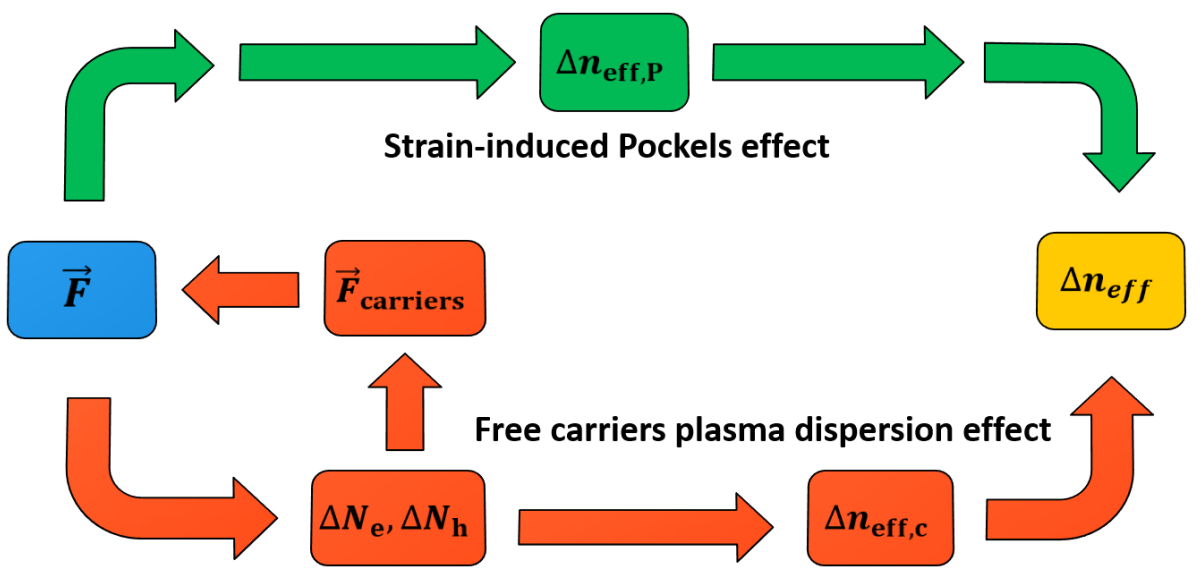

Figure 2. Schematic representation of the combination of strain induced Pockels effect and plasma dispersion effect.

Thereby, in order to study strain-induced Pockels effect, it is important to analyze the free carriers behavior and to find ways to eliminate, or at least reduce, their influence on the effective index change. To do so, we performed electro-optic amplitude modulation (the modulation amplitude being proportional to the effective index change) at frequencies higher than $5 \mathrm{GHz}$ since it has been shown that free carrier lifetime is of the order of $1 \mathrm{~ns}^{14}$ In this range, we can assume that the free carriers don't play a significant role in the modulation response.

\section{RESULTS}

We achieved electro-optic modulation on an integrated Mach-Zehnder interferometer (MZI). The sample consists in a $260 \mathrm{~nm}$ thick, $5 \mathrm{~mm}$ long silicon MZI on a $2 \mu \mathrm{m}$ thick buried oxide (BOX), stressed by a $700 \mathrm{~nm}$ thick SiN layer deposited via plasma enhanced chemical vapor deposition. The SiN film compressive stress is 1.3 GPa. Gold coplanar electrodes were deposited on top of the SiN film to apply the driving electric field in the waveguide. A schematic view of the cross section is shown on figure 3 .

High speed modulation experiments were performed. Frequencies higher than $5 \mathrm{GHz}$ were applied since at these frequencies the recombination/generation process are too slow to imply free carriers plasma dispersion effect. We obtained a bandwidth of approximately $10 \mathrm{GHz}$ (see figure 4). The cut-off frequency is due to a velocity mismatch between the driving electric field and the optical mode propagating in the structure.

At such frequencies, using a very lowly doped silicon substrate (residual p-doping impurities), we believe that the observed electro-optic modulation is due to strain-induced Pockels effect. Indeed, the experimental results 


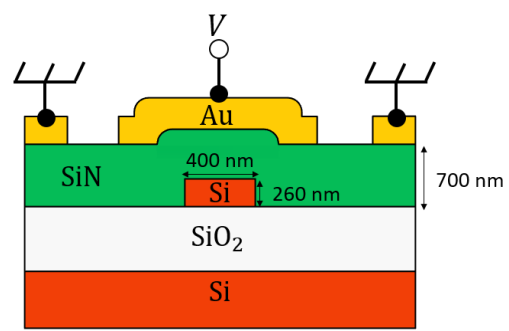

Figure 3. Schematic cross-section of the waveguide.

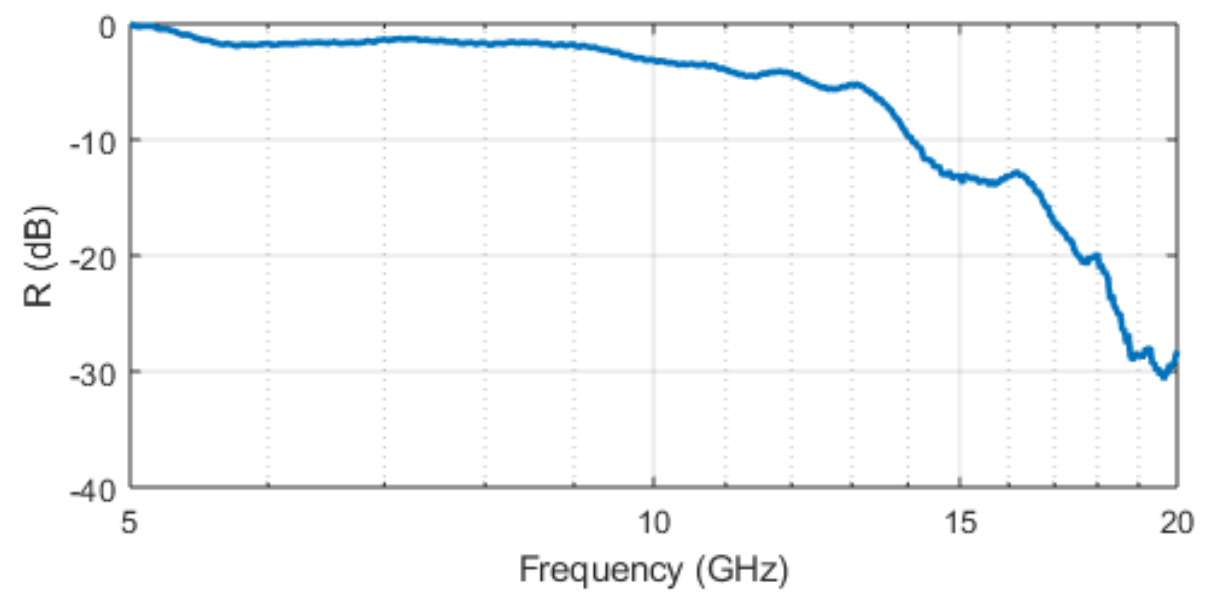

Figure 4. High speed experiment result (normalized).

showed no bias voltage dependence for frequencies higher than $3 \mathrm{GHz}$, while carriers distribution and electric field screening effect are strongly dependent on the bias voltage. This is a first clue that at these frequencies, the electro-optic modulation we observed is not based on plasma dispersion effect. Moreover, we performed the modulation on several tilted MZI with angles ranging from $0^{\circ}$ to $45^{\circ}$ according to the [011] direction. We observed a strong variation of the modulation efficiency as shown on figure 5. The carriers behavior is not expected to show such an angle dependence, which again points toward a Pockels effect based electro-optic modulation.
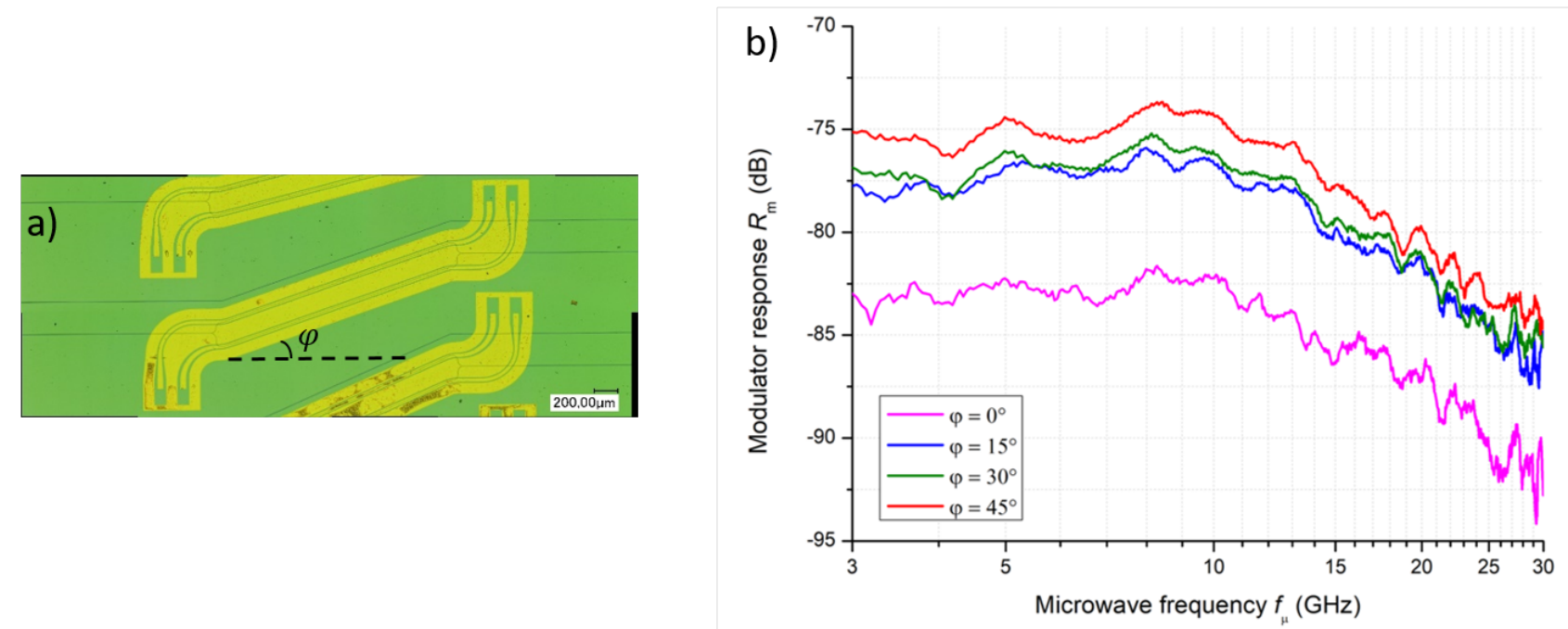

Figure 5. a) Optical image of a tilted MZI (top view). b) Angle dependent modulator response. 
a)
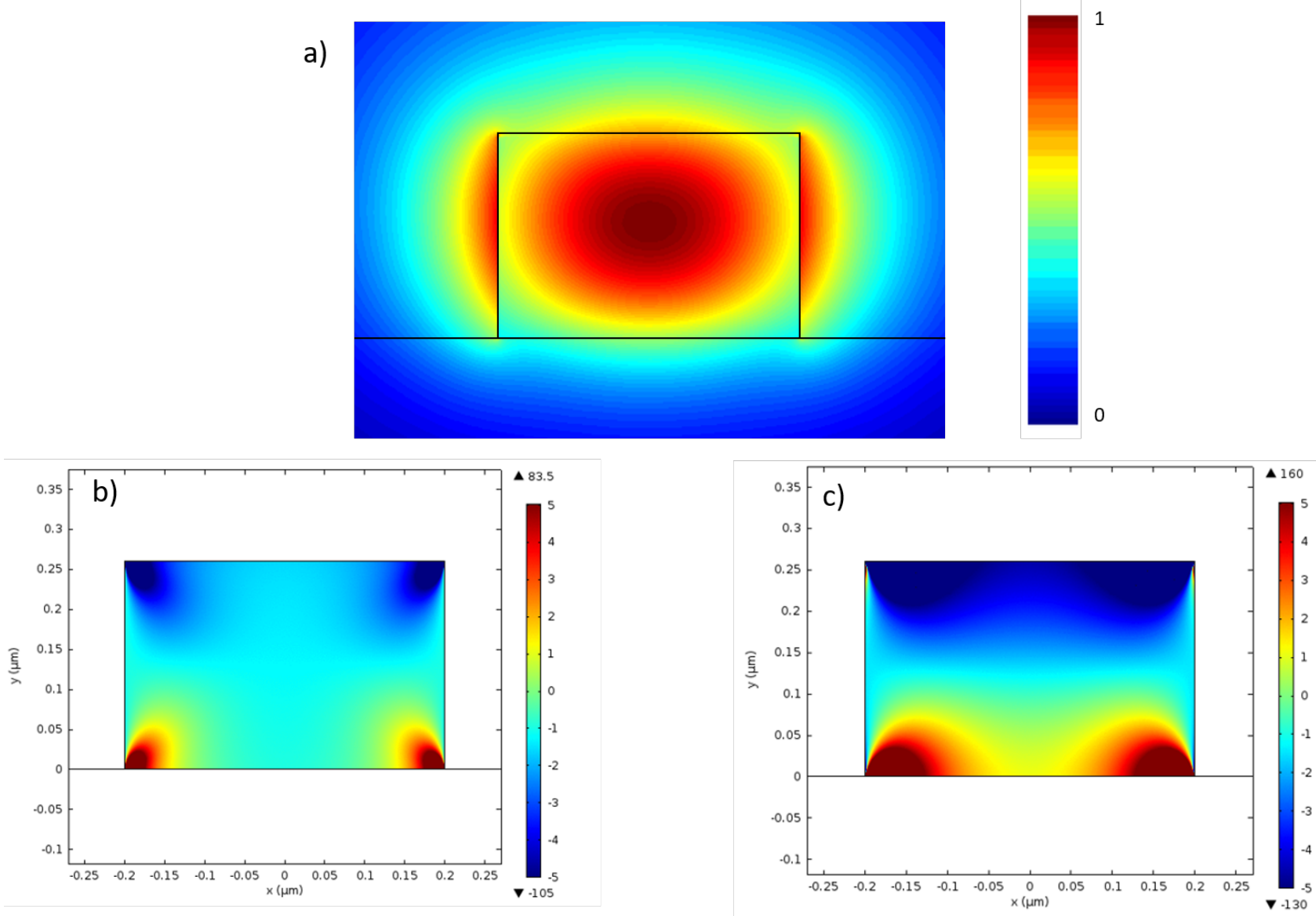

Figure 6. a) Distribution of the optical mode (normalized). b) Distribution of $\chi_{x x y}^{(2)}(\mathrm{pm} / \mathrm{V})$ in the waveguide cross section at $\varphi=0^{\circ}$. c) Distribution of $\chi_{x x y}^{(2)}(\mathrm{pm} / \mathrm{V})$ in the waveguide cross section at $\varphi=45^{\circ}$.

By fitting the curves of figure $5 \mathrm{~b}$ ), it was possible to determine the components of the $\Gamma$ tensor that participate to Pockels effect for a TE incident field. Then, the strain gradients, the optical mode and the driving electric field were numerically calculated in the waveguide cross section in order to calculate the second order susceptibility. It led to an effective value of $\left|\chi_{x x y}^{(2)}\right|$ ranging between $1.2 \mathrm{pm} / \mathrm{V}$ and $1.5 \mathrm{pm} / \mathrm{V}$, depending on the crystal orientation.

\section{DISCUSSION}

The effective $\chi^{(2)}$ we obtained is extremely low compared to LiNbO electro-optic modulator for example (several hundreds of $\mathrm{pm} / \mathrm{V}$ ), but it is the result of an overlap between the local $\chi^{(2)}$ distribution (directly linked to the strain gradient distribution) with the optical mode, and higher local values of $\simeq 100 \mathrm{pm} / \mathrm{V}$ are achieved on the edges of the waveguide (see figure 6). A way to improve the global modulation efficiency is then to increase the overlap between the strain gradients and the optical mode. Indeed, the high strain gradients are concentrated in the edges and the corners of the waveguide, while the optical mode is mostly distributed on the center of the waveguide, resulting in a poor overlap between the two.

Moreover, it would be interesting to investigate other ways to apply stress on the waveguide to obtain strain gradients close to the center of the waveguide. For example, I. Olivares et al. ${ }^{10}$ proposes to deposit two straining films with different stress on the device: one on the right half of the waveguide, one on the left one. This way, the discontinuity of stress would induce a strong strain gradient in the middle of the silicon waveguide, improving the effective $\chi^{(2)}$ of two orders of magnitude. The use of a different contact configuration can also help increasing the driving electric field in the structure. For example, the use of doped region in a p-i-n diode configuration can prevent from suffering of the electric field screening effect. 


\section{CONCLUSION}

In our study, we were able to experimentally demonstrate strain-induced Pockels effect based elctro-optic modulation in a silicon Mach-Zehnder interferometer stressed by a silicon nitride layer. To do so, we performed high speed measurements in order to exclude free carriers plasma dispersion effect. Our study showed that it is possible to locally obtain second order susceptibility values of the order of a hundred $\mathrm{pm} / \mathrm{V}$, which begins to be comparable of other materials used for electro-optic modulation. However, it is needed to improve the overlap between the susceptibility distribution and the optical mode distribution in the waveguide to increase the effective $\chi^{(2)}$ value. The next step to our study is to numerically investigate the strain-induced Pockels effect and the free carriers effect in order to improve our structure.

\section{REFERENCES}

[1] Fedeli, J. M., Di Cioccio, L., Marris-Morini, D., Vivien, L., Orobtchouk, R., Rojo-Romeo, P., Seassal, C., and Mandorlo, F., "Development of silicon photonics devices using microelectronic tools for the integration on top of a cmos wafer," Advances in Optical Technologies 2008, 1-15 (2008).

[2] Soref, R. and Bennett, B., "Electrooptical effects in silicon," IEEE Journal of Quantum Electronics 23, 123-129 (January 1987).

[3] Reed, G. T., Mashanovich, G., Gardes, F. Y., and Thomson, D. J., "Silicon optical modulators," Nature Photonics 4, 518-526 (Aug 2010).

[4] Vivien, L., Osmond, J., Fédéli, J.-M., Marris-Morini, D., Crozat, P., Damlencourt, J.-F., Cassan, E., Lecunff, Y., and Laval, S., "42 ghz pin germanium photodetector integrated in a silicon-on-insulator waveguide," Optics Express 17, 6252 (Apr 2009).

[5] Gosciniak, J. and Rasras, M., "High-bandwidth and high-responsivity waveguide-integrated plasmonic germanium photodetector," Journal of the Optical Society of America B 36, 2481 (Sep 2019).

[6] Chen, S., Li, W., Wu, J., Jiang, Q., Tang, M., Shutts, S., Elliott, S. N., Sobiesierski, A., Seeds, A. J., Ross, I., and et al., "Electrically pumped continuous-wave iii-v quantum dot lasers on silicon," Nature Photonics 10, 307-311 (May 2016).

[7] Zhang, J., Haq, B., O'Callaghan, J., Gocalinska, A., Pelucchi, E., Trindade, A. J., Corbett, B., Morthier, G., and Roelkens, G., "Transfer-printing-based integration of a iii-v-on-silicon distributed feedback laser," Optics Express 26, 8821 (Apr 2018).

[8] Damas, P., Marris-Morini, D., Cassan, E., and Vivien, L., "Bond orbital description of the strain-induced second-order optical susceptibility in silicon," Physical Review B 93, 165208 (Apr 2016).

[9] Damas, P., Berciano, M., Marcaud, G., Alonso Ramos, C., Marris-Morini, D., Cassan, E., and Vivien, L., "Comprehensive description of the electro-optic effects in strained silicon waveguides," Journal of Applied Physics 122, 153105 (Oct 2017).

[10] Olivares, I., Parra, J., Brimont, A., and Sanchis, P., "Enhancing pockels effect in strained silicon waveguides," Optics Express 27, 26882 (Sep 2019).

[11] Sharif Azadeh, S., Merget, F., Nezhad, M. P., and Witzens, J., "On the measurement of the pockels effect in strained silicon," Optics Letters 40, 1877 (Apr 2015).

[12] Olivares, I., Angelova, T., and Sanchis, P., "On the influence of interface charging dynamics and stressing conditions in strained silicon devices," Scientific Reports 7, 7241 (Dec 2017).

[13] Berciano, M., Marcaud, G., Damas, P., Le Roux, X., Crozat, P., Alonso Ramos, C., Pérez Galacho, D., Benedikovic, D., Marris-Morini, D., Cassan, E., and et al., "Fast linear electro-optic effect in a centrosymmetric semiconductor," Communications Physics 1, 64 (Dec 2018).

[14] Borghi, M., Mancinelli, M., Merget, F., Witzens, J., Bernard, M., Ghulinyan, M., Pucker, G., and Pavesi, L., "High-frequency electro-optic measurement of strained silicon racetrack resonators," Optics Letters 40, 5287 (Nov 2015). 